\section{Should the Postgraduate Year 2 (PGY-2) Residency Be Focused on Advanced Pharmacy Practice Training?}

\section{THE "PRO" SIDE}

For the past 2 years, the Canadian Pharmacy Residency Board (CPRB) has been working with stakeholders to create "advanced practice" (ACPR2) residency accreditation standards (where the professional designation ACPR means Accredited Canadian Pharmacy Residency). These advanced practice residencies are referred to by some, using the US nomenclature, as "postgraduate year 2 (PGY-2)" residencies, and the corresponding standards should be finalized by the end of calendar year 2015 .

In undertaking this process, the CPRB was compelled to answer 2 key questions:

- How would a second year of residency training provide greatest value in the new landscape of pharmacy training programs?

- How can demands from pharmacists and employers for "specialization" be balanced with demand from the profession for large numbers of practitioners capable of solving more complex problems and fulfilling more advanced practice roles both inside and outside of traditional specialty categories?

Fortunately, we believe, both questions are effectively answered by the approach now being taken. First, using the residency competency of "Provide patient care as a member of interprofessional teams" as an example, the "advanced" level means that residency program graduates will be required to demonstrate expert-level skill. ${ }^{1}$ This requirement distinguishes ACPR2 residencies from the existing ACPR residencies, where "proficient" is the required level of performance for this competency. Second, these higher performance levels must be demonstrated in the context of complex patients or complex therapy problems. The level of performance and the context complexity form the defining scaffold for the proposed advanced practice residency standards.

The shift in accreditation standards across health professions training programs, including pharmacy, to competency-based standards over the past 10 years means that the focus is now on skills (i.e., demonstration of practice skills at a defined level in a defined context of complexity), not knowledge. Within this framework, the focus is on developing higher levels of skill for solving more complex problems, rather than on acquiring more specialized knowledge. Attaining knowledge is an essential but insufficient element in the process of developing skills.
Specialization and advanced practice, though sometimes associated, are independent variables in professional education. Specialization does not correspond to a proficient or expert skill level. Furthermore, the "specialized" designation does not connote that the patients or their therapy problems are complex in nature, whereas the "advanced" designation does. For example, a pharmacist may be working in a specialized environment (e.g., HIV, pediatrics, geriatrics, or cardiology), offering care to a defined population, but that care may involve issues of low complexity that do not demand advanced skills. There may be a need for specialized knowledge associated with the practice, but the care itself could be highly protocolized, routine, and noncomplex. Attaining specialized knowledge can be accomplished with or without attaining advanced skills.

Recognizing this, and after much debate, the CPRB chose to delink the concepts of "advanced" and "specialized", making "advanced" the central construct of the ACPR2 standards. Specialization is embraced (as described in the section on "defined area of practice" within the standards) but is not a requirement for performing at an advanced level. Focusing on one area (i.e., specializing) may facilitate developing the skills and attitudes necessary to practise at an advanced level, but it is not necessary. What is necessary is performing at a high level while providing care in more complex situations and more complex patients. Focusing on "advanced" rather than "specialized" has many advantages, including the following:

- Advanced practice skills are more portable across patient populations. This approach demands that advanced residency graduates demonstrate expert-level skill in solving problems in complex patients, regardless of the defined area of practice in which that care is provided. We believe that acquiring this level of proficiency, for those who choose to pursue ACPR2 training, will serve graduates, their colleagues, and most importantly their patients and the public better than attaining highly specialized knowledge that is applied at less than an expert level. Such training will also provide a solid foundation for subsequently acquiring specialized skill and knowledge, should a pharmacist's career path demand it.

- Advanced practice promotes a rational continuum of patientcare training for pharmacists. Entry-to-practice PharmD programs impart competent-level skills in moderately complex patients, whereas ACPR residencies push performance to the proficient level. The proposed ACPR2 residencies will impart expert-level skills for the care of more complex patients, in alignment with the competency role domains of 
the CanMEDS Physician Competency Framework, the Association of Faculties of Pharmacy of Canada, and the CPRB across the spectrum of training.

- Advanced practice is simpler to establish, maintain, and administer. Unique sets of standards for each specialty area are not required, making the onerous process of wrestling to national consensus on criteria and competencies among every specialty group irrelevant. This issue has hampered the American College of Clinical Pharmacy in creating and managing specialization certifications, and the American Society of Health-System Pharmacists has been able to offer only a small number of practice-specific PGY-2 standards. A system of specialized ACPR2 standards would be unwieldy and costly to develop and maintain. Constant disciplinespecific changes would be required, and teams of specialized surveyors would be costly, if not impossible, to cultivate in our vast, sparsely populated country. These limitations would hinder the development of specialized programs and would be undesirable for existing specialization programs that may desire accreditation.

- Advanced practice is more flexible. Content experts can design the curriculum to reflect real-world practice needs, even organization-specific ones, guided by the standards, which articulate the level of performance required of residents.

- Advanced practice is more practical. Advanced practice performance is measurable and generic and is therefore a more rational basis for standards than specialization, with its vast diversity of required knowledge and specific skills.

- Advanced practice aligns better with the nature and future of the pharmacy profession. Unlike medicine, which has established specialties and subspecialties representing well-defined career paths that are, in many ways, industries unto themselves, pharmacy is evolving along different lines for different reasons. Primary care, ambulatory care, and community care are settings where advanced training to care for complex patients with a diverse array of therapeutic issues is increasingly required. Meanwhile, established inpatient-oriented specialties will be well served by ACPR2 programs and standards focused on advanced practice in these patient populations.

- Advanced practice promotes the rapid development of more ACPR2 programs. If the psychiatry community had to wait for specialized psychiatry residency standards to be developed before creating a psychiatry ACPR2 program, the system of residency training would likely fail to serve the patients and the profession to which it is directed. As proposed, any sufficiently advanced practice group can begin development of an ACPR2 program immediately.

Why not demand both specialized knowledge and expert level performance in complex patients? Our view is that such a pinnacle of training goes beyond what should be the minimum standard required for an ACPR2 program, which is, after all, only
1 year. Such a goal may become the domain of fellowship programs following ACPR2. Time will tell.

Advanced practice standards do not prevent trainees from acquiring specialized knowledge and skills during the residency. Rather, they explicitly embrace the defined area of practice where the learning may be focused. In addition, they adhere to the principle of performing at higher levels in the context of more complex patients as the central rubric for measuring success of the resident and the program.

For these reasons, centring the next major step in the development of pharmacy practice residency programs in Canada on advanced practice is the right move for residents, care teams, and, most importantly, patients.

Reference

1. Dreyfus SE. The five-stage model of adult skill acquisition. Bull Sci Technol Soc. 2004;24(3):177-81.

Peter Loewen, BSc(Pharm), PharmD

Faculty of Pharmaceutical Sciences University of British Columbia

Vancouver, British Columbia

Allan Mills, BSc(Pharm), PharmD

Pharmacy Department

Trillium Health Partners

Mississauga, Ontario

Curtis Harder, BSC(Pharm), PharmD

Department of Pharmacy

Vancouver Island Health Authority

Victoria, British Columbia

Competing interests: The authors are members of the Canadian Pharmacy Residency Board and of its Standards Committee, responsible for the development and maintenance of pharmacy practice residency standards in Canada. The opinions expressed here are those of the authors, and not necessarily those of the Canadian Society of Hospital Pharmacists, the Canadian Pharmacy Residency Board, or its Standards Committee.

\section{THE "CON" SIDE}

Sometime in the not-too-distant future, a graduate of an advanced year-2 pharmacy practice residency program (ACPR2) will be attending a job interview. Let's listen in ...

INTERVIEWER: Thanks for agreeing to this interview. Would you describe the qualifications you hold that make you the best candidate for this job?

INTERVIEWEE: Absolutely. I'm a pharmacist, and I have completed an accredited pharmacy practice residency, as well as an advanced year-2 pharmacy practice residency. (private musing: Oh please, know what that is ...) INTERVIEWER: Advanced practice residency . . . would that be like a fellowship? (Hmmm . . others I've interviewed have also had a residency to prepare for advanced practice roles, and it didn't take them 2 years...) 
INTERVIEWEE: Yes, that is a good analogy. I did a year-2 residency in pediatrics, so it was focused training in that clinical practice setting.

INTERVIEWER: (What on earth is "focused" training?)

So then ... you spent an additional year specializing in pediatrics?

INTERVIEWEE: Yes and no. I spent a year learning advanced clinical skills in pediatrics. So I'm specialized in the sense that I have acquired a breadth of pediatric pharmacotherapy expertise. Technically speaking, I didn't complete a "specialized residency" and Canada doesn't officially recognize "specialist" titles in pharmacy, so I can't claim that level of qualification. (I hate explaining this... I can barely make sense of it myself. . .)

INTERVIEWER: So, how has that "advanced but not specialty—or fellowship—-training in pediatrics" prepared you for this role? (Does she know she's got 15 other questions to complete within the 40 minutes left in this interview?)

INTERVIEWEE: I've had an extra year of training with expert clinicians, refining my patient care skills to provide safe, effective, collaborative care to individual pediatric patients. I've also taken on a leadership role to improve quality of care for groups of patients. (It would have been so much simpler to say that I completed a specialized residency in pediatrics...)

We certainly respect the deep thoughts that generated the international competency and credentialing models referred to by our esteemed colleagues. ${ }^{1,2}$ Those models use complex "education-speak" and lovely graphics to differentiate "advanced practice" from "specialization" in pharmacy, but to patients, care teams, and health care administrators, isn't this just a matter of semantics? To the nonpharmacist eye, the Canadian Pharmacy Residency Board's definition of "advanced practice" and the Blueprint for Pharmacy ${ }^{3}$ working definition of "pharmacist specialists" are nearly identical. Both imply that the pharmacist has skills beyond those of an entry-to-practice pharmacist. Ability to deal with complexity is what differentiates entry-to-practice pharmacy care providers from those with advanced practice generalist or specialist skills. The practice area can be either broad or narrow in scope, and the only discernible difference between a specialty-trained pharmacist and an advanced practice generalist pharmacist is that graduates of a specialty residency would be prepared for and expected to challenge a specialist certification exam.

In interview rooms, employers evaluate candidates for a position in which they will invest at least $\$ 1.8$ million (2015 dollars) in salary, benefits, and training amortized over 10 years, in the hope of hiring a pharmacist with the highest level of skill to deliver disease prevention and care to groups of similar patients in a safe and efficient way. That's what most employers would call a "specialist" pharmacist. Why would an employer invest in an advanced generalist for a nephrology practice if she could hire a nephrology specialist pharmacist, fully knowing that this pharmacist has a breadth of knowledge in dealing with patients who have renal disease? It just so happens that Canada's hospital employers, in a needs assessment about specialization in pharmacy, have already indicated a willingness to invest their money in specialist pharmacists. ${ }^{3}$ Hospital pharmacy job descriptions have included the word "specialist" for more than 35 years—a fact that both authors (admittedly a generation apart in experience!) have encountered when searching for employment. For years, this meant having a second professional degree in pharmacy, or an Accredited Canadian Pharmacy Residency (ACPR) with additional practice experience involving a defined patient population or disease subspecialty. With changes in university-based programs, increasingly the challenge these days is figuring out whether the "specialist" claim on an applicant's curriculum vitae will be matched by the talent needed in practice.

The needs assessment also revealed a big challenge for hospital care. ${ }^{3}$ Pharmacy in Canada is deeply divided on the issue of specialization, and a fix isn't imminent. So, as we graduate the last of the old-style PharmDs, a source of replacement for those original PharmDs is needed, especially before all of them permanently exchange their parkas for snowbird status. What we also need in the "short" term-arguably the next 20-30 years at the current rate of practice change in pharmacy-is a reliable way to identify who has acquired and refined the knowledge, skills, and ability to look after patients with complex care issues in actual practice. It would be a bonus if those practitioners have also learned enough about the big picture to have shown the ability to improve care for whole groups of complex patients in a collaborative way. It would be a double bonus, but not required, if they had proof from a Canadian-made certification exam saying they are "real" specialists.

There is one thing we know for sure in hospital settings: the ACPR is the mark of an advanced generalist pharmacotherapy practitioner, but additional practice in a focused area is needed to hone specialist skill and knowledge. The market has sought pharmacy specialists, yet we are now going to graduate residents with "focused but not specialist advanced practice" training? Does it really add value to create an ACPR2 graduate whose claim to fame is, at best, being "a more advanced generalist"? If we go down this path, will we have missed the most important opportunity to come along in Canadian hospital pharmacy in a very long while? We think so.

ACPR residencies are highly respected and valued training programs that have been a trusted brand for 60 years. Canada already produces specialized residency graduates and has done so for years-it's just that those programs are not accredited (raising questions about quality and comparability of training) and are producing a very small number of graduates. ${ }^{4}$ Specialized residency training programs of the future represent a major 
opportunity to confirm specialist "talent" even in the absence of a Canadian-made specialist certification process. So, can we get over ourselves, our education-speak, and our lovely graphical models? Can we get down to the real business of saying what we've been expecting in hospital pharmacy in Canada for 35 years? The term is "specialist", not "ACPR2". Let's back up our commitment to patients by growing Canada's next generation of specialists through specialized residencies. Let's be the early adopters in Canada so that 20-30 years from now, we'll be ready with as many as 200 specialty residency graduates for that first "made in Canada" specialist credentialing exam.

\section{References}

1. Advanced Pharmacy Practice Framework Steering Committee (APPFSC). An advanced pharmacy practice framework for Australia. Deakin West, ACT (Australia): Pharmaceutical Society of Australia; 2012 [cited 2015 Sep 3]. Available from: http://advancedpharmacypractice.com.au/download/ framework/advanced-pharmacy-practice-framework.pdf

2. Blair MM, Freitag RT, Keller DL, Kiser TH, Marrs JC, McGivney MS, et al. Proposed revision to the existing specialty and specialist certification framework for pharmacy practitioners [AACP White Paper]. Pharmacotherapy. 2009; 29(4):489.

3. Needs assessment of specialization in pharmacy in Canada. Ottawa (ON): Canadian Pharmacists Association; 2015 [cited 2015 Sep 3]. Available from: http://199.103.61.199/docs/resource-items/needs-assessment-of-specializationin-pharmacy-in-canada---final-report.pdf

4. Sheehan N. Pharmacy specialty residency programs in Canada: present and future considerations [PowerPoint presentation]. Université de Montréal; 2010 [cited 2015 Sep 3]. Available from: https://www.afpc.info/sites/default/ files/Sheehan_specialty_res_AFPC.pdf

\section{Ashley N Walus, BScPharm, ACPR}

Donna M M Woloschuk, PharmD

Pharmacy Practice Residency Program

Winnipeg Regional Health Authority

Winnipeg, Manitoba

Competing interests: Donna Woloschuk is President-Elect of the Canadian Council for Accreditation of Pharmacy Programs, is a former Chairperson of the Canadian Pharmacy Residency Board, and is a Director of a pharmacy practice residency program. The opinions expressed in this article are solely those of the authors for the purpose of debate. They do not necessarily represent the views of the Canadian Council for Accreditation of Pharmacy Programs, the Canadian Society of Hospital Pharmacists Manitoba Branch, the University of Manitoba College of Pharmacy, or the Winnipeg Regional Health Authority, with whom the authors are affiliated.

\section{Compounding: Guidelines for Pharmacies 2014}

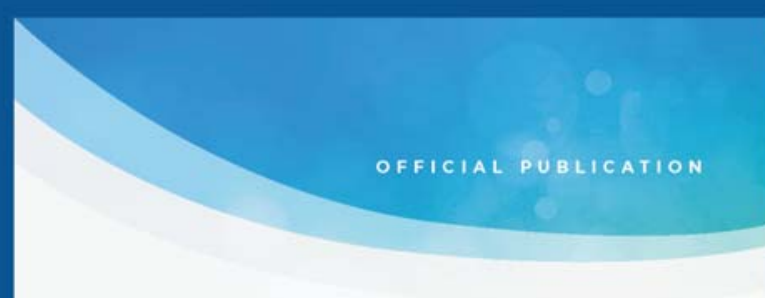

Compounding: Guidelines for Pharmacies

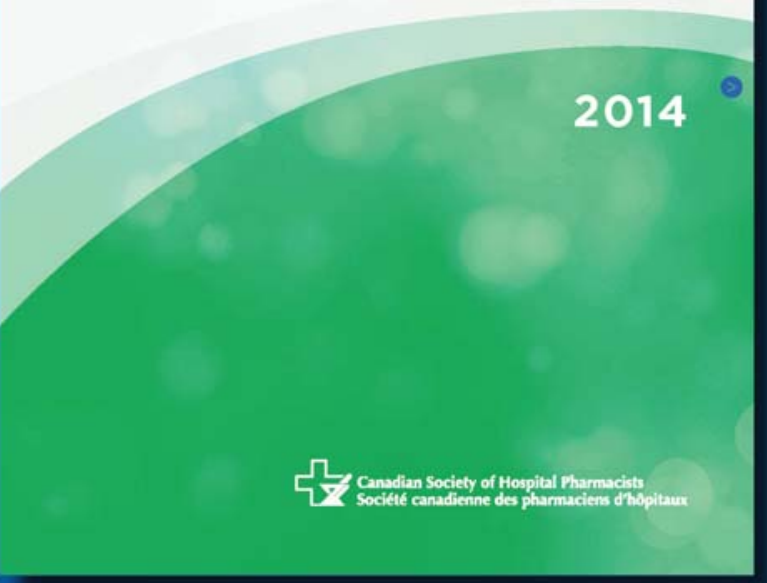

The Canadian Society of Hospital Pharmacists' comprehensive set of guidelines covers the compounding in pharmacies whenever compounded preparations are intended for human use, regardless of the route of administration or whether the preparation is related to research purposes. These guidelines also apply to the preparation of radiopharmaceuticals and other hazardous pharmaceuticals.

Find our order form here: http://www. cshp.ca/dms/dmsView/I CSHP-Publications-form-Jan2015.pdf

Contact the CSHP Publications Administrator for more information:

E: aiannaccio@cshp.ca P: 613-736-9733 x:228 